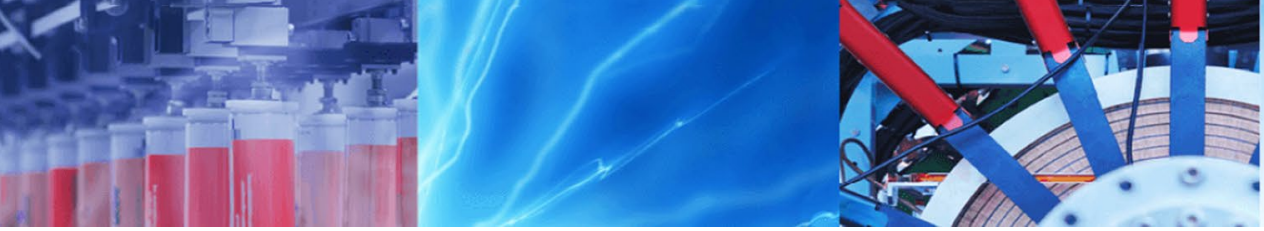

Research Article

\title{
Computational evaluation of a novel beta radiation probe design using integrated circuits
}

\author{
Jeremy W. King ${ }^{1} \cdot$ Manit D. Shah ${ }^{2} \cdot$ Craig M. Marianno $^{1}$ (D) Sunil P. Khatri ${ }^{3} \cdot$ Sunil S. Chirayath $^{1}$
}

Received: 14 July 2020 / Accepted: 16 February 2021 / Published online: 27 February 2021

(C) The Author(s) 2021 OPEN

\begin{abstract}
Researchers at Texas A\&M University (TAMU) have designed the radiation integrated circuit (RIC) for deployment as a new radiation detection system. Most integrated circuits are susceptible to radiation-induced failures, and decades of research have gone into solving this problem. Research at TAMU has led to a novel integrated circuit design that utilizes both radiation-hardened areas (RHAs) and radiation-sensitive areas (RSAs) to take advantage of these failures. The RSAs are susceptible to charged particle interactions, allowing the RIC to detect alpha and beta particles. However, beta particles are more penetrating compared to alpha particles, resulting in a lower interaction probability for beta particles incident on a bare RIC. In any material, the higher the beta energy, the deeper the beta particle can penetrate; therefore, the use of a wedge-shaped attenuator for beta particle detection not only increases interaction probability, but also provides the capability to perform maximum beta energy discrimination in the field. The objective of this research was to optimize the design of the RIC. Monte Carlo N-particle radiation transport code (MCNP) simulations assessed the beta particle detection and maximum energy discrimination performance of plate glass, borosilicate (Pyrex ${ }^{\circledR}$ ) glass, acrylic (Lucite ${ }^{\circledast}$, and natural rubber attenuators. In this proof-of-concept analysis, natural rubber was observed to be the optimal attenuating material for the beta probe with respect to maximum energy discrimination capability and weight, but all materials considered proved to be good candidates. The results of this study are promising and indicate the potential to achieve maximum beta particle energy discrimination of $50 \mathrm{keV}$ using a wedged, natural rubber attenuator on the RIC.
\end{abstract}

Keywords Beta particle detection · Beta particle spectroscopy · Instrumentation · survey probe

\section{Introduction}

\subsection{Background}

With the miniaturization of semiconductor technology, circuits on chips have become increasingly sensitive to radiation damage and, therefore, more likely not to function properly in a high radiation field. Researchers at Texas A\&M University (TAMU) have been able to convert this failure into a feature with the radiation integrated circuit (RIC). The RIC contains both radiation-hardened areas (RHAs) and radiation-sensitive areas (RSAs) [1]. Figure 1 provides a simple overview of the RIC design. The RSAs contain electrical circuits which are sensitive to charged particle interactions $[2,3]$. A single RHA, designed to be highly resistant to radiation effects, monitors multiple RSAs for changes in voltage, indicative of a charged particle interaction $[2,3]$. If an RHA registers no voltage change in a particular RSA, the RHA moves on to observe the next RSA in the chain. This cycle continues until a voltage change is detected in an RSA. An RSA that suffers a charged particle interaction will recover to its normal state within several nanoseconds,

Craig M. Marianno, marianno@tamu.edu| 'Department of Nuclear Engineering, Texas A\&M University, College Station, TX 77840, USA. ${ }^{2}$ Canadian Nuclear Laboratories, Chalk River, ON, Canada. ${ }^{3}$ Department of Electrical and Computer Engineering, Texas A\&M University, College Station, USA. 


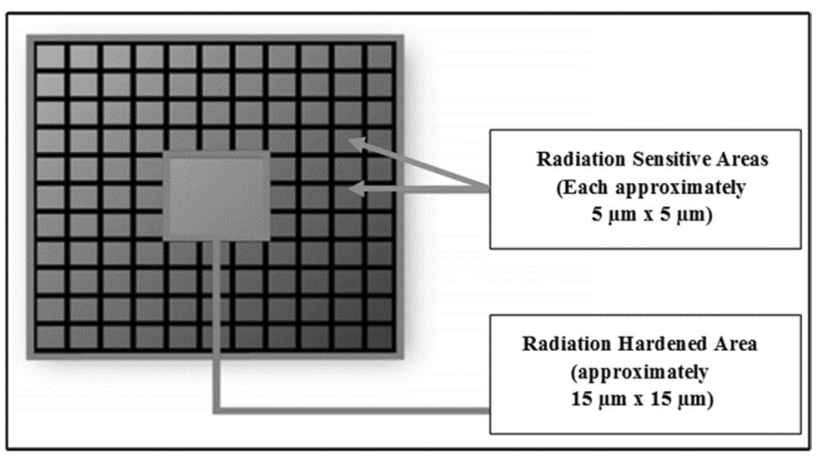

Fig. 1 A conceptual illustration of the RIC design. A single RHA monitors multiple RSAs for charged particle interactions

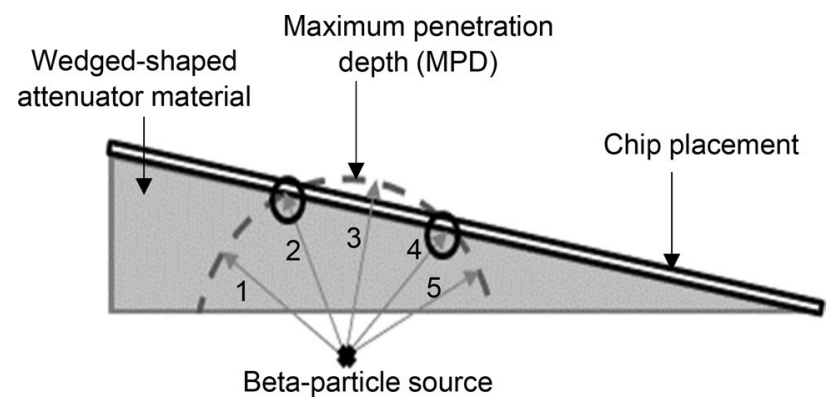

Fig. 2 Illustration of maximum beta particle penetration distance in a wedge-shaped attenuator, the phenomenon by which the RIC can perform maximum energy discrimination

allowing an RHA to monitor about 40 RSAs, given a worstcase internal clock frequency of $1 \mathrm{GHz}$.

Hundreds of thousands $\left(\sim 10^{5}\right)$ of RSAs can be fabricated on a $1 \times 1 \mathrm{~cm} \mathrm{RIC,} \mathrm{comprising} \mathrm{about} 75 \%$ of the chip area $[1,3]$. Tens of thousands $\left(\sim 10^{4}\right)$ of RHAs consume about $15 \%$ of the chip area, and the remainder of the RIC is used to implement signal processing and clocking circuits. The distribution of the total active area into many distinct, active regions renders the likelihood of multiple particle strikes in a single region between resets very low, especially in the low activity fields a survey probe of this type would be deployed in. As such, the system dead time is expected to be negligible. Survey probes utilizing the RIC can offer several advantages over current systems: reduced power consumption, reduced weight, relatively low cost when produced in bulk, and significantly smaller packaging because detection and signal processing are handled on a single chip [1-3].

\subsection{Working principle of the probe}

With RSAs sensitive to charged particles, RICs can be employed to detect beta particles. However, beta particles are far more penetrating than alpha particles, reducing the probability of a beta particle interaction with a bare RIC. Utilizing a wedge-shaped attenuator (Fig. 2) for beta particle detection not only increases the interaction probability, but also provides the capability to perform maximum beta energy $\left(\mathrm{E}_{\beta, \max }\right)$ discrimination in the field. Beta particle penetration (range) in matter is a function of the particle's energy and the material's properties. For a survey probe comprised of RICs, the attenuator thickness over each sector of the chip will be known, allowing the determination of $E_{\beta, \max }$ for beta contamination in the field. When the probe is fabricated, RICs will be placed on the inclined surface of the wedge such that beta particles will penetrate varying lengths of attenuator before arriving at the RIC surface.

Figure 2 exhibits the working principle used to determine $E_{\beta, \max }$. In the figure, a beta particle source is depicted below the probe base, shown along with the wedge-shaped attenuator. A beta particle of known energy has a known maximum penetration depth (MPD) in any given material. In the figure, an arc is shown which represents the MPD in the material. The arrowed lines indicate the tracks of five identical beta particles, labeled 1 through 5 , that strike the probe at different angles. For beta particles 1 and 5 , the attenuator thickness is greater than the MPD. These particles will not reach the chip. For beta particle 3 , the attenuator thickness is less than the MPD. This particle, which will penetrate the wedge, can either pass through the chip without affecting an RSA or interact in the chip. For beta particles 2 and 4, the attenuator thickness is slightly less than the MPD in the material. These particles will be near the end of their track and have minimal energy near the RIC surface, providing the highest probability of interaction. For this research, areas on the RIC where the attenuator thickness is slightly less than the MPD are jointly referred to as the region of interest (ROI). This $\mathrm{ROI}$ is a function of the beta particle source position along the probe, $E_{\beta, \max }$ and the attenuating material. Analyses of different attenuator materials were focused on interactions in the ROI, as explained in the next section.

It is important to note that the scope of this research is only to introduce a novel beta particle detector design and to explore through simulation how it could potentially be used to indicate the energy of the beta particles it registers. This research does not address the challenges of manufacturing or operational use in the field such as more complex source geometries, the presence of multiple source isotopes, and the added complexity of energy discrimination given the continuous energy spectrum of beta decay. The simulation work presented in this paper was used as a basis for creating the RIC which has since been fabricated. The challenges of manufacturing 
a finalized design and field use in complex beta radiation fields will be addressed in follow-on research.

\section{Methodology}

\subsection{Materials and geometry}

Simulations of the RIC were carried out using Monte Carlo N-particle radiation transport code, MCNP6.1 [4]. In these simulations, the attenuator material for the beta survey probe was modeled as a wedge. Four low atomic number (low-Z) materials were considered in this research: borosilicate (Pyrex $\left.{ }^{\circledR}\right)$ glass, plate glass, acrylic (Lucite ${ }^{\circledR}$ ), and natural rubber. The isotopics of the materials were acquired from Pacific Northwest National Laboratory's Compendium of Material Composition Data for Radiation Transport Modeling, henceforth referred to as The Compendium [5]. Isotope ZAID (atomic number, Z, and atomic mass, A, identification) values, weight fractions, and the density of each material are summarized in Table 1. Several simulations were carried out for each attenuator material to assess its energy discrimination capability by varying the source position and $E_{\beta, \max }$.

As a handheld survey instrument, a probe comprised of RICs must balance energy discrimination capability, physical size, and weight. Low-Z materials were selected due to their superior beta particle stopping power in the target energy regime and low density. For low incident energies (less than about $20 \mathrm{MeV}$ ) like those considered in this study, beta particles lose energy more rapidly in low-Z materials due to their higher collision rate compared to high-Z materials [6]. It is only at higher incident energies that the superior energy reduction per collision of high-Z materials dominates; therefore, beta particles have shorter range in low-Z materials within the energy regime considered. Probe weight was an especially significant concern which is why only very lightweight, organic materials were ultimately selected. Organic materials are known to degrade (yellowing, loss of elasticity, etc.) when irradiated to sufficient doses. For example, many polymers and elastomers for the medical field are considered radiation compatible up to doses on the order of tens of kGy [7]. This is several orders of magnitude greater than the annual dose limit to radiation workers in the United States of 5000 mrem or 0.05 Gy [8]. Radiation fields intense enough to accumulate to degrading doses within a timeframe that would bring the probe's utility into question would also prevent human entry. Additionally, field instruments of the type envisioned are characterized at regular intervals to check for performance changes. As such, material degradation was not considered in this investigation.

Table 2 lists the modeled dimensions for each attenuator material. The $\mathrm{w}, \mathrm{l}$, and $\mathrm{h}$ values represent the width, length, and height (maximum thickness) of the different attenuators as shown in Fig. 3. For example, the plate glass attenuator was $5 \mathrm{~cm}$ long by $5 \mathrm{~cm}$ wide, with a thickness that increased linearly along the length from 0 to $0.5 \mathrm{~cm}$. For each material, the attenuator dimensions
Table 1 Compendium reference number, isotopic composition, and density for each material used in the simulations

\begin{tabular}{|c|c|c|c|}
\hline Material (\# in the compendium) & ZAID & Weight fraction & Density $\left(\mathrm{g} \mathrm{cm}^{-3}\right)$ \\
\hline \multirow[t]{4}{*}{ Dry air (\#4) } & 06000 & 0.000124 & 0.001205 \\
\hline & 07000 & 0.755268 & \\
\hline & 08000 & 0.231781 & \\
\hline & 18000 & 0.012827 & \\
\hline \multirow[t]{6}{*}{ Borosilicate (Pyrex ${ }^{\oplus}$ ) glass (\#143) } & 05000 & 0.040064 & 2.23 \\
\hline & 08000 & 0.539562 & \\
\hline & 11000 & 0.028191 & \\
\hline & 13000 & 0.011644 & \\
\hline & 14000 & 0.377220 & \\
\hline & 19000 & 0.003321 & \\
\hline \multirow[t]{4}{*}{ Plate glass (\#146) } & 08000 & 0.459800 & 2.40 \\
\hline & 11000 & 0.096441 & \\
\hline & 14000 & 0.336553 & \\
\hline & 20000 & 0.107205 & \\
\hline \multirow[t]{3}{*}{ Acrylic (Lucite $\left.{ }^{\circledast}\right)(\# 182)$} & 01000 & 0.080538 & 1.19 \\
\hline & 06000 & 0.599848 & \\
\hline & 08000 & 0.319614 & \\
\hline \multirow[t]{2}{*}{ Natural rubber (\#272) } & 01000 & 0.118371 & 0.92 \\
\hline & 06000 & 0.881629 & \\
\hline
\end{tabular}


Table 2 Modeled dimensions of the wedge-shaped attenuator for each material

\begin{tabular}{llll}
\hline Attenuator material & $\begin{array}{l}\text { Width, w } \\
\text { (X-axis) } \\
{[\mathrm{cm}]}\end{array}$ & $\begin{array}{l}\text { Length, } \\
\text { I(Y-axis) } \\
{[\mathrm{cm}]}\end{array}$ & $\begin{array}{l}\text { Height, } \mathrm{h} \\
(\mathrm{Z} \text {-axis) }[\mathrm{cm}]\end{array}$ \\
\hline Borosilicate $\left(\right.$ Pyrex $^{\oplus}$ ) glass & 5.0 & 5.0 & 0.5 \\
Plate glass & 5.0 & 5.0 & 0.5 \\
Acrylic (Lucite ${ }^{\circledast}$ ) & 5.0 & 7.0 & 1.0 \\
Natural rubber & 5.0 & 7.0 & 1.1 \\
\hline
\end{tabular}

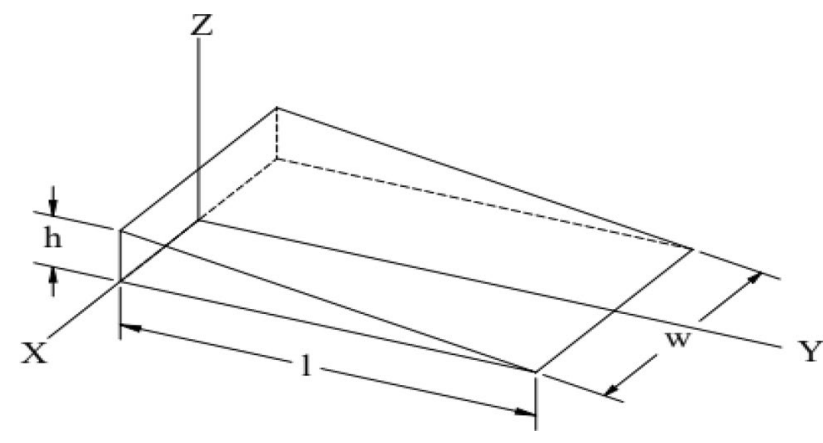

Fig. 3 A reference coordinate system for the beta probe attenuator model

were selected to allow $E_{\beta, \max }$ discrimination of beta particles with energies in excess of $2.1 \mathrm{MeV}$. For each attenuator material, the linear stopping power for beta particles varies, so the attenuators' dimensions were selected accordingly assuming linear penetration, which is a rough approximation used in working problems [6].

\subsection{MCNP simulations}

A surface flux detector (tally type F2 per MCNP code nomenclature) was created along the inclined surface of the wedge, centered around the Y-axis. Tally segments (the FS card in MCNP) were utilized to split the detector (tally) into many bins of interest. Each bin had a width along the $X$-axis of $0.1 \mathrm{~cm}$ and a length along the $Y$-axis of $0.1 \mathrm{~cm}$, resulting in a bin area slightly greater than $1 \mathrm{~mm}^{2}$ on the inclined surface. Such an area on the RIC chip contains several thousand RSAs. These bins lay flush to one another such that a $5 \mathrm{~cm}$ long probe model has 50 tally bins along its inclined surface.

Simulations were carried out to evaluate the $E_{\beta, \max }$ discrimination capability of each attenuator material. For this assessment, the initial energy of beta particles was varied, and the position of the source was varied accordingly. For each material, an isotropic, monoenergetic beta particle point source was simulated with energies ranging from 1.95 to $2.10 \mathrm{MeV}$ in increments of $0.01 \mathrm{MeV}$.
In each simulation, the point source was placed $1 \mathrm{~cm}$ below the attenuator $(Z=-1 \mathrm{~cm})$ and centered along the $X$-axis $(X=0 \mathrm{~cm})$. The source was also positioned along the $Y$-axis such that the attenuator thickness directly above the source equaled the material's MPD for the beta particle energy under consideration. MPD was calculated with the empirical formula given by Eq. 1 [6]. This empirical calculation of the MPD (Turner [6] uses the nomenclature, "range") assumes the continuous slowing down approximation (CSDA) and "straight-ahead travel." The result is an overestimation of electron penetration, which will shift the peak electron fluence rate along the inclined surface in the positive- $Y$ direction where the attenuator is less thick. Since the purpose of the study is to observe separation of the peaks at different energies, not to locate peaks precisely, these assumptions are acceptable for this research so long as the shift of expected peak positions is consistent across the energy range considered. The monoenergetic beta energy in the above simulations represents the maximum energy of all beta particles emitted. In reality, however, beta decay is a three-body problem, resulting in a continuous energy spectrum for beta particles. This spectrum can be predicted by the Fermi theory [9].

$M P D=\frac{1}{\rho} \begin{cases}0.412 E^{1.27-0.0954 \ln E} & 0.01 \leq E \leq 2.5 \\ 0.530 E-0.106 & E>2.5\end{cases}$

MPD: maximum particle depth [cm].

$\rho$ : material density $\left[\mathrm{g} \mathrm{cm}^{-3}\right]$.

$\mathrm{E}$ : electron (beta particle) energy [MeV].

On a manufactured probe utilizing RICs, energy will be indicated by the position of the particle fluence rate peak along the detector surface, so energy discrimination can be measured by the spacing of peaks of different energies along the surface. The location of the fluence rate peak for each energy in the 1.95 to $2.10 \mathrm{MeV}$ range was located, and the spatial energy resolution of the detector was calculated as the negative ratio of the difference of maximum and minimum energies to the difference of maximum and minimum peak locations along the $\mathrm{Y}$-axis as depicted in Eq. (2). The negative ratio is required because the peak position of the maximum energy is less than that of the minimum energy in the chosen coordinate system. By this formulation, a lower value of spatial energy resolution indicates better energy discrimination capability because there is greater distance between the peaks. 
$r=-\frac{E_{\max }-E_{\min }}{Y_{\max }-Y_{\min }}$

$r$ : spatial energy resolution $\left[\mathrm{MeV} \mathrm{cm}^{-1}\right]$.

$\mathrm{E}_{\mathrm{i}}$ : electron (beta particle) energy $[\mathrm{MeV}]$.

$Y_{i}$ : peak position of energy, $i[\mathrm{~cm}]$.

\section{Results and discussion}

In MCNP6.1, plate glass, borosilicate (Pyrex ${ }^{\oplus}$ ) glass, acrylic (Lucite $\left.{ }^{\circledR}\right)$, and natural rubber were simulated as wedgeshaped attenuators to characterize the maximum beta energy discrimination performance of a RIC-based survey instrument. In each simulation, the number of beta particles traversing the attenuator and eventually entering RSAs of the RIC was tallied. The analysis of the tallies focused on the ROIs for each attenuator.

\subsection{Plate glass}

The fluence rate tallies as a function of scoring bin position along the $\mathrm{Y}$-axis are presented in Fig. 4. Vertical error bars are displayed on the data points, but most are not visible because they are very small $(<1 \%)$. For clarity, only the results for initial energies of $1.95,2.00,2.05$, and 2.10 MeV are plotted. No tally multipliers were applied, so the results are representative of a $1 \mathrm{~Bq}$ source, hence the low fluence rate magnitudes. The relative errors of most data points were well under $10 \%$, satisfying the statistical check for a surface detector (F2 tally) in MCNP. Some tallies on the thickest portion of the detector $(0 \mathrm{~cm} \leq \mathrm{Y} \leq 0.5 \mathrm{~cm})$ had relative errors in excess of $10 \%$. The fluence rates in this region are very low, so the error bars are not visible.

Fluence Rate v. Position - Plate Glass

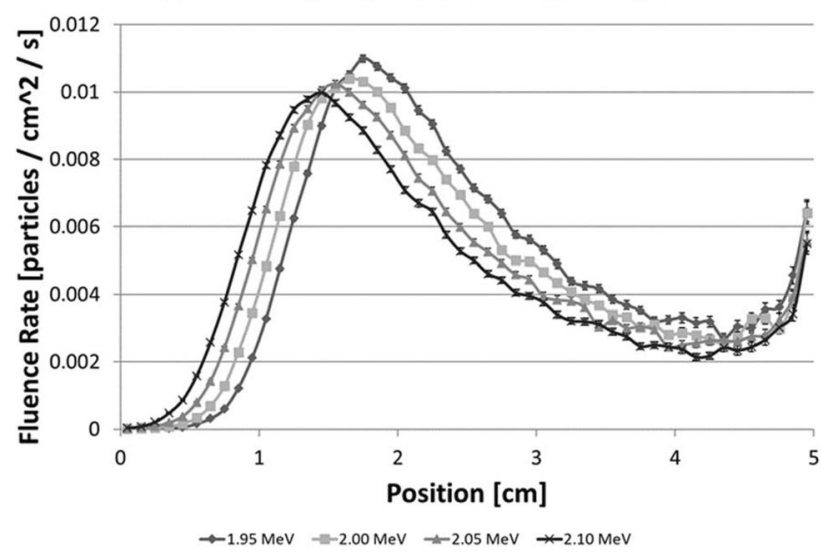

Fig. 4 A plot of the beta particle tallies of all scoring regions along the length of the probe with a plate glass attenuator
However, this region is isolated from the nearest energy peak studied in this research by about $1 \mathrm{~cm}$, so the slightly high relative errors are not of concern. A connecting line is shown to highlight the trend of the results. The trend in the data is dominated by the attenuation of beta particles in the material: higher energy peaks occur on the left where the material is thicker, and lower energy peaks occur on the right where the material is thinner. To the left of the peaks, most particles do not penetrate all the way through the thicker portion of the material, and as a result, the fluence rate values are much lower. To the immediate right of the peaks, the beta particles can penetrate the attenuator and be tallied, but further to the right the total transport distance increases, reducing the fluence rate. To the far right of the peaks, the MPD becomes increasingly greater than the attenuation thickness, so the particles pass all the way through the material, resulting in increased fluence rate. This general behavior is illustrated in Fig. 5, which depicts the relationship between MPD and the linear travel distance of a particle. The empirical MPD formulation of Eq. 1 was used to calculate the MPD plotted in Fig. 5, slightly overestimating the MPD. The increasing fluence rate to the far right of the peaks is a result of simulation setup and would not be observed in a probe. The surface detector (MCNP F2 tally) was implemented to allow simplified problem geometry and reduce computation time. However, the F2 tally also scores higher energy beta particles passing through the thinner portions of the material rather than only lower energy particles expected to interact with the RIC. The result is an overestimation of particle interactions to the far right of the peaks. This follows from the fact that the thickness of attenuator material traversed by particles in this region is much less than the

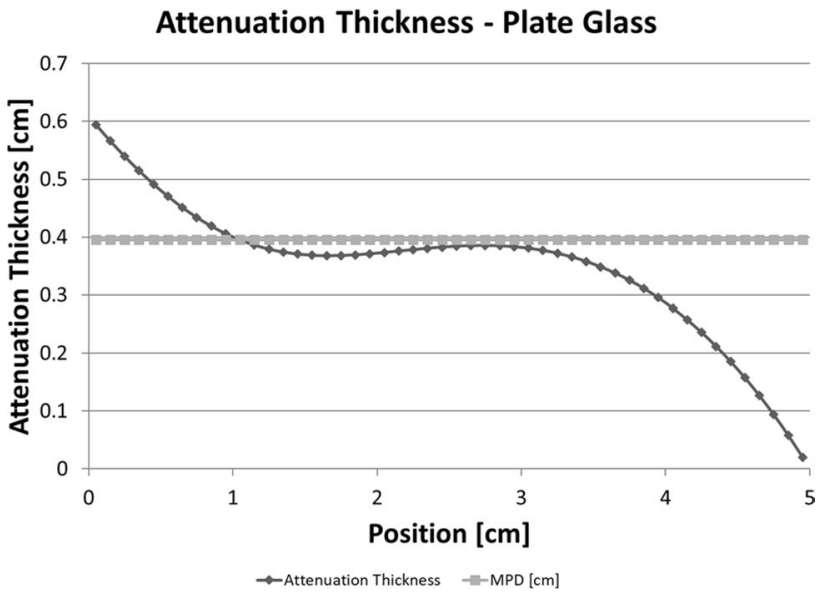

Fig. 5 The attenuation thickness that a beta particle traverses between the source and the inclined surface of the plate glass attenuator as a function of bin position on the $Y$-axis. This plot is for a $2 \mathrm{MeV}$ source placed at $(X, Y, Z)=(0,1.046,-1) \mathrm{cm}$ 
Fluence Rate v. Position - Plate Glass

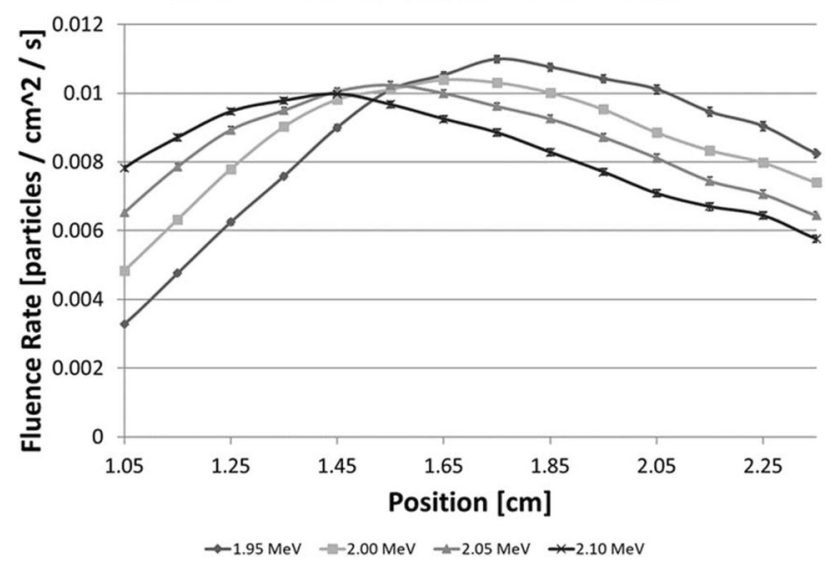

Fig. 6 A plot of the beta particle tallies of all scoring regions in the $\mathrm{ROI}$ of the probe with a plate glass attenuator

MPD as calculated from the CSDA. Since the focus of this research is the separation of peaks, this is an acceptable discrepancy but must be taken into account if mathematical models are to be developed describing the detector response.

The MPD for a $2 \mathrm{MeV}$ beta particle in plate glass is $0.395 \mathrm{~cm}$. From $1.05 \mathrm{~cm}$ along the probe length all the way to $5.00 \mathrm{~cm}$, the attenuator thickness for a beta particle emitted from a source at $(X, Y, Z)=(0,1.046,-1) \mathrm{cm}$ is less than the MPD (Fig. 5). However, between $1.05 \mathrm{~cm}$ and $2.35 \mathrm{~cm}$ along the probe length, the attenuator thickness is only slightly less than the MPD of a $2 \mathrm{MeV}$ beta particle. After traversing this distance, the beta particles are at the end of their track and within an energy range that can more readily interact in the RIC RSA. Similar trends were observed for all beta energies simulated.

For the plate glass attenuator, the $\mathrm{ROI}$ is between 1.05 and $2.35 \mathrm{~cm}$. The defined probe region encompasses fluence rate maxima for all initial beta energies from $1.95 \mathrm{MeV}$ to $2.10 \mathrm{MeV}$. Figure 6 displays the same data plotted in Fig. 4, focusing on the ROI $(1.05-2.35 \mathrm{~cm})$. In the ROI, the trend for the attenuator thickness was convex upward as a function of the probe length (Fig. 5). In this region, there is a local minimum attenuator thickness which corresponds to the scoring bin containing the maximum number of counts. For the $2 \mathrm{MeV}$ beta particles, the peak count was estimated across the tally area at $1.75 \mathrm{~cm}$ into the probe length. The closer look provided by Fig. 6 reveals that each energy peak occurred within a distinct tally bin, critical for energy discrimination.

\section{Fluence Rate v. Position - Borosilicate Glass}

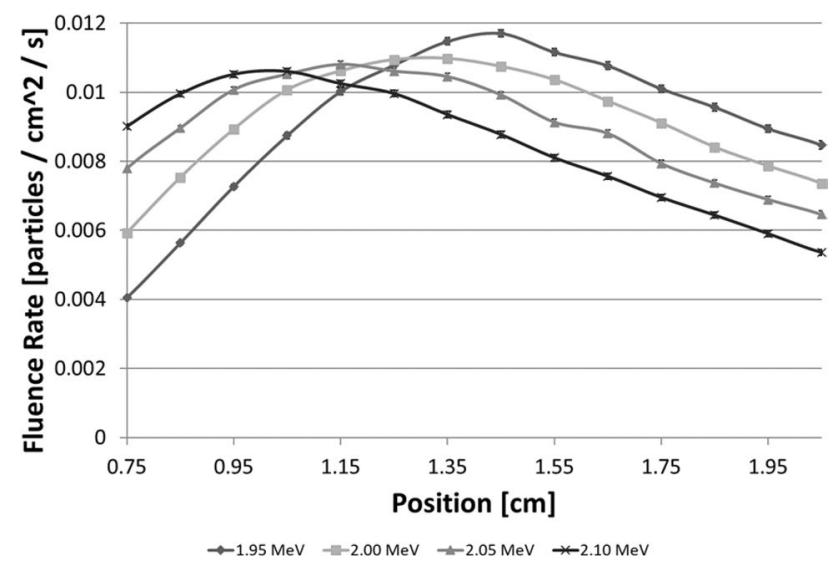

Fig. 7 A plot of the beta particle tallies of all scoring regions in the $\mathrm{ROI}$ of the probe with a borosilicate glass attenuator

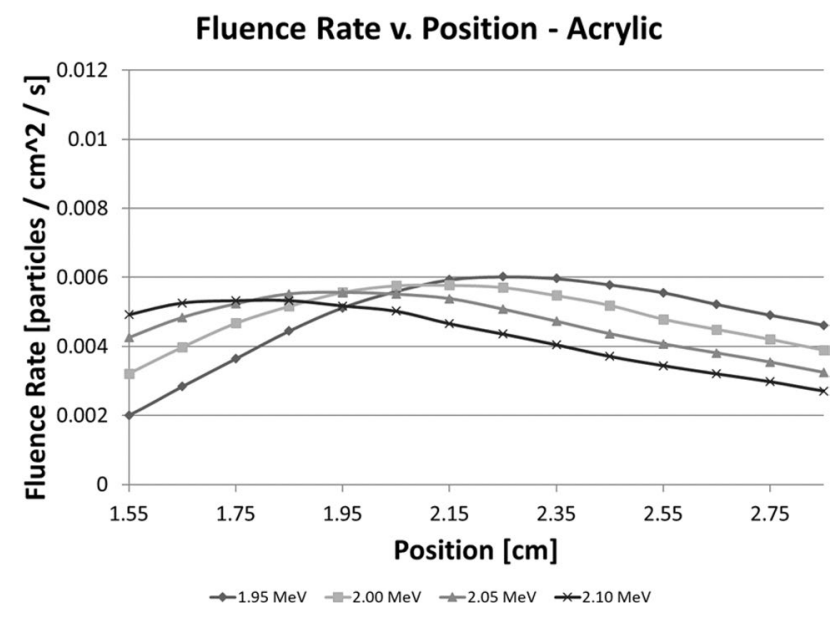

Fig. 8 A plot of the beta particle tallies of all scoring regions in the $\mathrm{ROI}$ of the probe with an acrylic attenuator

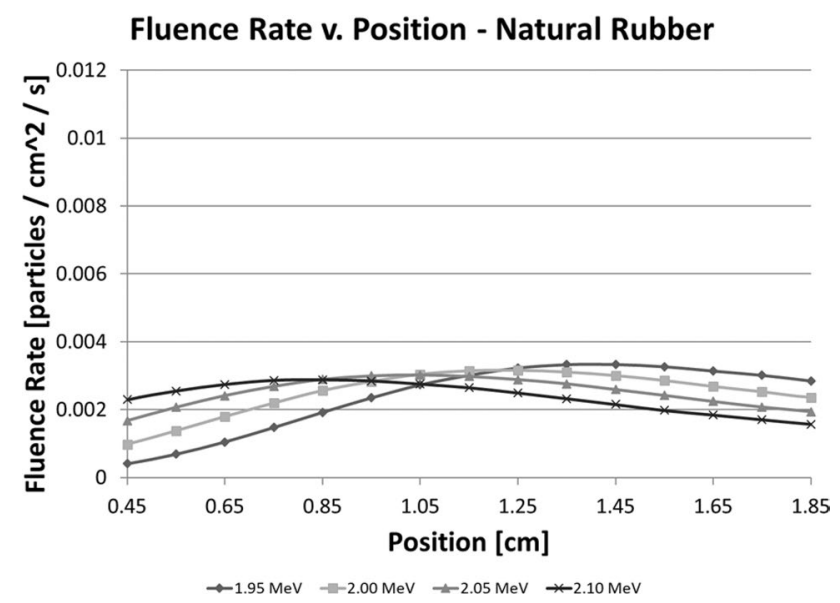

Fig. 9 A plot of the beta particle tallies of all scoring regions in the $\mathrm{ROI}$ of the probe with a natural rubber attenuator 
Table 3 The spatial energy resolution, mass, and effective atomic and mass numbers of all attenuator materials

\begin{tabular}{lllll}
\hline & $\mathrm{r}\left[\mathrm{MeV} \mathrm{cm}^{-1}\right]$ & Mass [g] & $\mathrm{Z}_{\text {eff }}$ & $\mathrm{A}_{\text {eff }}$ \\
\hline Plate glass & 0.500 & 15.0 & 11.60 & 23.32 \\
Borosilicate (Pyrex $^{\oplus}$ ) glass & 0.375 & 13.9 & 10.32 & 20.75 \\
Acrylic (Lucite $^{\oplus}$ ) & 0.375 & 20.8 & 06.24 & 12.40 \\
Natural rubber & 0.250 & 17.7 & 05.41 & 10.74 \\
\hline
\end{tabular}

\subsection{Discrimination analysis}

Figures 7, 8, and 9 display the number of beta particles tallied in the ROIs of borosilicate (Pyrex ${ }^{\circledast}$ ) glass, acrylic $\left(\right.$ Lucite $^{\circledR}$ ), and natural rubber, respectively. The ROIs (with respect to the $2 \mathrm{MeV}$ reference) were $0.75-2.05 \mathrm{~cm}$ for Pyrex $^{\circledR}$ glass, $1.55-2.85 \mathrm{~cm}$ for Lucite ${ }^{\circledR}$, and $0.45-1.85 \mathrm{~cm}$ for natural rubber. Analogous to Fig. 6 for plate glass, the plotted values are the beta particle fluence rate tallied in each bin $\left[\mathrm{cm}^{-2} \mathrm{~s}^{-1}\right]$, presented as functions of the initial beta particle energy and distance along the probe length. The trends in these figures are similar to the trend observed in Fig. 6 with the key difference being the MPD of the beta particles in each material. The MPD for $2 \mathrm{MeV}$ beta particles is $0.426 \mathrm{~cm}, 0.798 \mathrm{~cm}$, and $1.032 \mathrm{~cm}$ in Pyrex ${ }^{\circledR}$ glass, Lucite $^{\circledR}$, and natural rubber, respectively.

The energy peaks in the plate glass (Fig. 6) were all separated by $0.1 \mathrm{~cm}$, a single tally bin in the chosen discretization. The energy peaks in the borosilicate glass (Fig. 7) and acrylic (Fig. 8) were separated by $0.1-0.2 \mathrm{~cm}$ ( 1 or 2 bins). The energy peaks in the natural rubber (Fig. 9) were all separated by $0.2 \mathrm{~cm}$ ( 2 bins).

Table 3 summarizes the energy discrimination performance and mass for each attenuator material. All materials investigated performed comparably, but natural rubber was observed to show the best spatial energy resolution (lowest energy change per unit distance, $0.25 \mathrm{MeV} \mathrm{cm}^{-1}$ ). The better energy resolution for natural rubber can be explained by its low effective atomic number (5.41) and effective mass number (10.71) compared to the other attenuator materials. For low initial particle energies (less than $20 \mathrm{MeV}$ ) like those considered in this investigation, beta particles lose energy more rapidly in materials of low atomic number due to the higher collision rate compared to high-Z materials [6]. The substantial hydrogen content of natural rubber reduces the effective atomic number and enhances the attenuation of beta particles in the material. Additionally, with a range of 13.9-20.8 g, all attenuators had similarly low masses. Natural rubber performed the best in these simulations, but all four materials investigated are good candidates for a RIC survey probe. Since the beta particle count drives the signal generated in the $\mathrm{RSA}$, a large difference in counts from one position to the

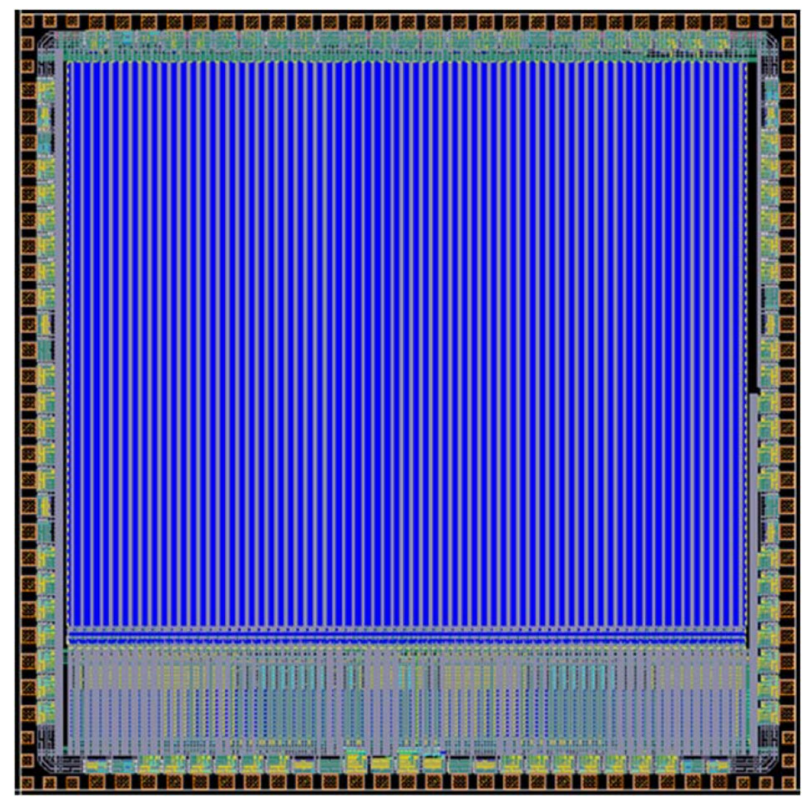

Fig. 10 X-ray image of the individual detection elements within the RIC

next along an attenuator makes energy discrimination easier to perform.

This calculated energy resolution serves as an estimator of hardware performance. The manufactured RIC features 70 distinct detection elements arranged parallel to one another, 52 of which can be monitored individually (Fig. 10). Hardware limitations and design priorities prevent all 70 elements from being monitored individually. For simplicity, if the 52 monitorable elements are presumed to be spaced evenly along the $1 \mathrm{~cm}$ width of the $\mathrm{RIC}$, the RIC has a resolution of about $4.8 \mathrm{keV}$ per element as given by the spatial energy resolution of $250 \mathrm{keV} \mathrm{cm}^{-1}$ for a natural rubber attenuator. If two tally bins (about 10 detection elements) of spacing between peaks are required for reliable peak discrimination-the worst-case scenario in the simulations-then $E_{\beta, \max }$ discrimination to about $50 \mathrm{keV}$ may be possible using a natural rubber wedged attenuator.

The natural rubber wedge design was $7 \mathrm{~cm}$ in length and $5 \mathrm{~cm}$ in width and the height increased linearly from 0 to $1.1 \mathrm{~cm}$ (Table 2). This design can measure $\mathrm{E}_{\beta, \max }$ in excess of $2.1 \mathrm{MeV}$. The range of $\mathrm{E}_{\beta, \max }$ detection can be extended by simply increasing the length and height. Conversely, an extremely compact design option is achievable but will reduce the range of measurable $E_{\beta, \max }$ values. For example, to detect $E_{\beta, \max }$ in the range of 0.5 to $2.1 \mathrm{MeV}$, the wedge height (thickness) would necessarily range from 0.18 to $1.1 \mathrm{~cm}$ and the length would be reduced to $5.87 \mathrm{~cm}$. 


\section{Conclusions}

The RIC RSAs are sensitive to charged particles, allowing them to detect alpha particles and beta particles. Beta particle detection with the RIC is more challenging because they are more penetrating than alpha particles and are less likely to interact on the bare integrated circuit. In order to optimize beta particle detection, attenuator materials can be placed on a RIC to reduce incident energy so that the probability of interaction is increased. Another advantage of using an attenuator is that the maximum depth a beta particle can penetrate is a function of its incident energy and the material properties. In this research, MCNP6.1 simulations were carried out to investigate the use of wedge-shaped attenuators made of plate glass, borosilicate (Pyrex ${ }^{\circledast}$ ) glass, acrylic (Lucite ${ }^{\circledast}$ ), and natural rubber for $E_{\beta, \max }$ discrimination. If energy discrimination is achievable, survey probes made of RICs will have considerable advantages over existing in situ beta particle detection technology.

To evaluate the energy discrimination capability of each material, a spatial energy resolution was calculated. This ratio reflects the spatial separation of energy. The finer the spatial energy resolution, the better the $\mathrm{E}_{\beta, \max }$ discrimination capability. The natural rubber wedged attenuator had the best spatial energy resolution of $0.25 \mathrm{MeV} \mathrm{cm}^{-1}$ compared to $0.375,0.375$, and $0.5 \mathrm{MeV} \mathrm{cm}^{-1}$ for Pyrex ${ }^{\oplus}$, Lucite $^{\circledast}$, and plate glass, respectively. However, these values are comparable. Likewise, the masses of the attenuators made of each material were comparable, ranging from 13.9-20.8 g. As such, each material investigated is a good candidate for a RIC survey probe. The calculated spatial energy resolution serves as an estimator of energy discrimination capability of the RIC. Thereby, there exists a potential to perform $E_{\beta, \max }$ discrimination within $50 \mathrm{keV}$ using a natural rubber wedged attenuator.

For this research, only a wedge-shaped attenuator was used to investigate the possibility of $E_{\beta, \max }$ discrimination. The authors acknowledge that other attenuator geometries may be more efficient and such possibilities will be investigated in future work.

\section{Compliance with ethical standards}

Conflict of interest The authors have no conflict of interest to declare.

Open Access This article is licensed under a Creative Commons Attribution 4.0 International License, which permits use, sharing, adaptation, distribution and reproduction in any medium or format, as long as you give appropriate credit to the original author(s) and the source, provide a link to the Creative Commons licence, and indicate if changes were made. The images or other third party material in this article are included in the article's Creative Commons licence, unless indicated otherwise in a credit line to the material. If material is not included in the article's Creative Commons licence and your intended use is not permitted by statutory regulation or exceeds the permitted use, you will need to obtain permission directly from the copyright holder. To view a copy of this licence, visit http://creativecommons .org/licenses/by/4.0\%.

\section{References}

1. Marianno C, Khatri S (2011) ARI-MA: A Low-Cost Integrated Circuit Based Detector for Gamma and Neutron Detection. Proposal to the National Science Foundation

2. Garg R, Jayakumar N, Khatri S (2006) A Design Approach for Radiation-hard Digital Electronics, in: Proc. 43rd ACM/IEEE Design Automation Conference DAC, San Francisco, CA, United States, 773-778.

3. Garg R (2009) Analysis and Design of Resilient VLSI Circuits, Dissertation. Texas A\&M University, Texas

4. Pelowitz DB et al. (2013) MCNP User's Manual Version 1.0, Los Alamos National Laboratory report no. LA-CP-13-00634

5. McConn RJ Jr., Gesh CJ, Pagh RT, Rucker RA, Williams III RG (2011) Compendium of Material Composition Data for Radiation Transport Modeling, Pacific Northwest National Laboratory report no. PNNL-15870 Rev.

6. Turner J (2007) Atoms, Radiation, and Radiation Protection, 3rd edn. Wiley-VCH Verlag GmbH \& Co. KGaA, Weinheim

7. "Material Consideration: Radiation Processing." Sterigenics, n.d. 1/28/21. https://sterigenics.com/wp-content/uploads/2018/09/ Material-Considerations-Irradiation-Processing_WEB-v3.pdf.

8. United States Nuclear Regulatory Commission (2018) "Subpart C-Occupational Dose Limits," August 24. https://nrc.gov/readi ng-rm/doc-collections/cfr/part020/part020-1201.html.

9. Krane K (1987) Introductory nuclear physics, 3rd edn. Wiley, New York

Publisher's Note Springer Nature remains neutral with regard to jurisdictional claims in published maps and institutional affiliations. 